\title{
Indigenous Becoming: Genesis and Resonance of Selve, a Play co-written with Sylvana Opoya from Taluhwen, Guiana, Amazonia
}

\section{Le devenir autochtone : Genèse et résonance de Selve, une pièce de théâtre co-écrite avec Sylvana Opoya depuis Taluhwen, Guyane, Amazonie}

\author{
Christophe Rulhes
}

Writer, Theatre Director and Anthropologist, France

\begin{abstract}
This article describes Sylvana Opoya's contribution to the writing and staging of Selve, a play performed and designed in France by Christophe Rulhes and the GdRA. In the text of the play, Sylvana, a 22-year-old Wayana woman in the French Guiana Amazon, talks about pollution and gold panning. Via video extracts from interviews, her uncle Aimawale Opoya, the tipatakem or village chief of Taluhwen, addresses the question of Wayana territorial sovereignty in relation to "white people's" ecological thought. Selve is inspired by the Amazon rainforest of Sylvana and Aimawale, and the remote Occitan language and peasant traditions of Quercy Rouergue (in Aveyron, France), the homeland of author and director Christophe Rulhes. As a result, various ways of wanting to belong to earth are echoing in Selve. In this article, the artist Christophe Rulhes uses methods from anthropology (he graduated from the Ecole des Hautes Etudes en Sciences Sociales) to underline the direct influence of Wayana's native culture on Selve's conception.
\end{abstract}

\section{Résumé}

L'article expose la contribution de Sylvana Opoya à l'écriture et à la mise en scène de Selve, une pièce de théâtre jouée et conçue en France par Christophe Rulhes et le GdRA. Dans le texte de la pièce, Sylvana, jeune femme Wayana de 22 ans en Amazonie guyanaise française, traite de la pollution et de l'orpaillage. Via des extraits vidéos d'entretiens, son oncle Aimawale Opoya, tipatakem ou chef du village de Taluhwen, parle quant à lui de la question de la souveraineté territoriale des Wayana, en rapport à la pensée écologique " des Blancs ». De la forêt amazonienne de Sylvana et d'Aimawale aux confins du Quercy Rouergue occitan, oral et paysan, de l'auteur et metteur en scène Christophe Rulhes, s'entend peutêtre dans Selve un possible écho entre diverses façons de vouloir appartenir à la terre. Dans cet article, Christophe Rulhes, artiste utilisant les méthodes de l'anthropologie dont il est diplômé de l'Ecole des Hautes Etudes en Sciences Sociales, souligne l'influence directe de l'autochtonie wayana sur la conception de Selve.

Keywords: Theatre, French Guiana, Amazonia, Ecology, Occitanie

eTropic: electronic journal of studies in the tropics publishes new research from arts, humanities, social sciences and allied fields on the variety and interrelatedness of nature, culture, and society in the tropics. Published by James Cook University, a leading research institution on critical issues facing the worlds' Tropics. Free open access, Scopus, Google Scholar, DOAJ, Crossref, Ulrich's, SHERPA/RoMEO, Pandora, ISSN 1448-2940. Creative Commons CC BY 4.0. Articles are free to download, save and reproduce. Citation: to cite this article include Author(s), title, eTropic, volume, issue, year, pages and DOI: http://dx.doi.org/10.25120/etropic.19.1.2020.3732 


\section{Introduction}

$\mathrm{E}$ ntre 2017 et 2019, l'équipe d'artistes du GdRA ${ }^{1}$ s'est rendue en Guyane pour créer la pièce Selve ${ }^{2}$. II s'agissait de fabriquer du théâtre et du récit à partir d'enquêtes menées sur ce territoire hyper-divers en faune, flore, langues. La création de Selve faisait suite à celle de Lenga, une proposition invitant sur scène Lizo James, Xhosa d'Afrique du Sud au Cap, et Maheriniaina Pierre Ranaivoson, Merina de Madagascar à Antananarivo. Les textes de Lenga et de Selve, destinés au théâtre mais issus d'enquêtes de terrain, forment un diptyque publié aux éditions des Solitaires Intempestifs et intitulé La Guerre des Natures, dans lequel des contributions autochtones témoignent directement de la pluralité et de la fragilité des langues sur des territoires à très forts enjeux environnementaux (Rulhes \& le GdRA, 2019). Des problèmes liés à l'habitat, l'extractivisme, la déforestation, la gestion des déchets, la brutale modification de la subsistance, frappent tant les forêts de Madagascar ou de Guyane que les townships du Cap ou les villages récents des rives amazoniennes françaises. Se jouent aussi en ces paysages des drames et des félicités intergénérationnelles liés à l'apprentissage des langues et des pratiques culturelles. Lenga et Selve jalonnent un chemin d'écriture dont le point de départ est mon expérience propre de la perte linguistique, terrienne et paysagère, dans un contexte de langue familiale orale, paysanne et occitane. L'apparition du glyphosate s'y est accompagnée des grammaires de normalisation de la langue et de l'abandon partiel d'une pensée de la terre.

Cette contribution traite donc du processus d'écriture à quatre mains du texte de Selve par Sylvana Opoya et moi, Christophe Rulhes, auteur, metteur en scène, anthropologue et musicien au sein du GdRA. II rend compte du parcours de vie de la principale autrice, femme engagée, petite-fille d'un chaman ou pïjai apalaï et nièce d'un oncle qui se définit comme métis wayana. L'article s'ouvre avec une évocation biographique de Sylvana. La deuxième partie cite largement les paroles de cette jeune femme et d'Aimawale, frère de sa mère Jolanda, chef coutumier du village. Ils parlent notamment de l'orpaillage et de la pollution au mercure. Enfin, une troisième partie en forme d'ouverture évoque un écho possible entre deux appartenances à la terre, celle des Amérindiens du village de Taluhwen, en Guyane française, sur la rive droite du haut Maroni qui marque la frontière avec le Suriname où vivent aussi les Wayana, et celle de ma famille paysanne du Quercy-Rouergue occitan, où là aussi, des enjeux de territoire et de langues traversent des corps et des personnes.

\footnotetext{
${ }^{1}$ Groupement de Recherche Artistique que j'ai formé en 2005 avec Julien Cassier alors jeune diplômé du Centre Nationale des Arts du Cirque, et Sébastien Barrier, comédien. J'étais alors doctorant anthropologue, chercheur allocataire associé au Centre d'Anthropologie de l'EHESS de Toulouse.

${ }^{2}$ Etaient présents le chorégraphe Julien Cassier qui co-anime le GdRA avec moi, la photographe Hélène Canaud, le dessinateur Benoît Bonnemaison-Fitte, le documentariste Nicolas Pradal, le régisseur David Løchen.
} 
L'écriture de Selve est le résultat de deux temps de terrain et résidence dans le village amazonien de Taluhwen et de cinq séjours communs en métropole et en Suisse, où l'autrice a suivi la mise en scène de ses mots. Elle a pleinement pu participer à la conception de l'œuvre théâtrale. Sur cette vidéo, on peut voir un montage des premières répétitions de Sylvana pour Selve : https://vimeo.com/314022751

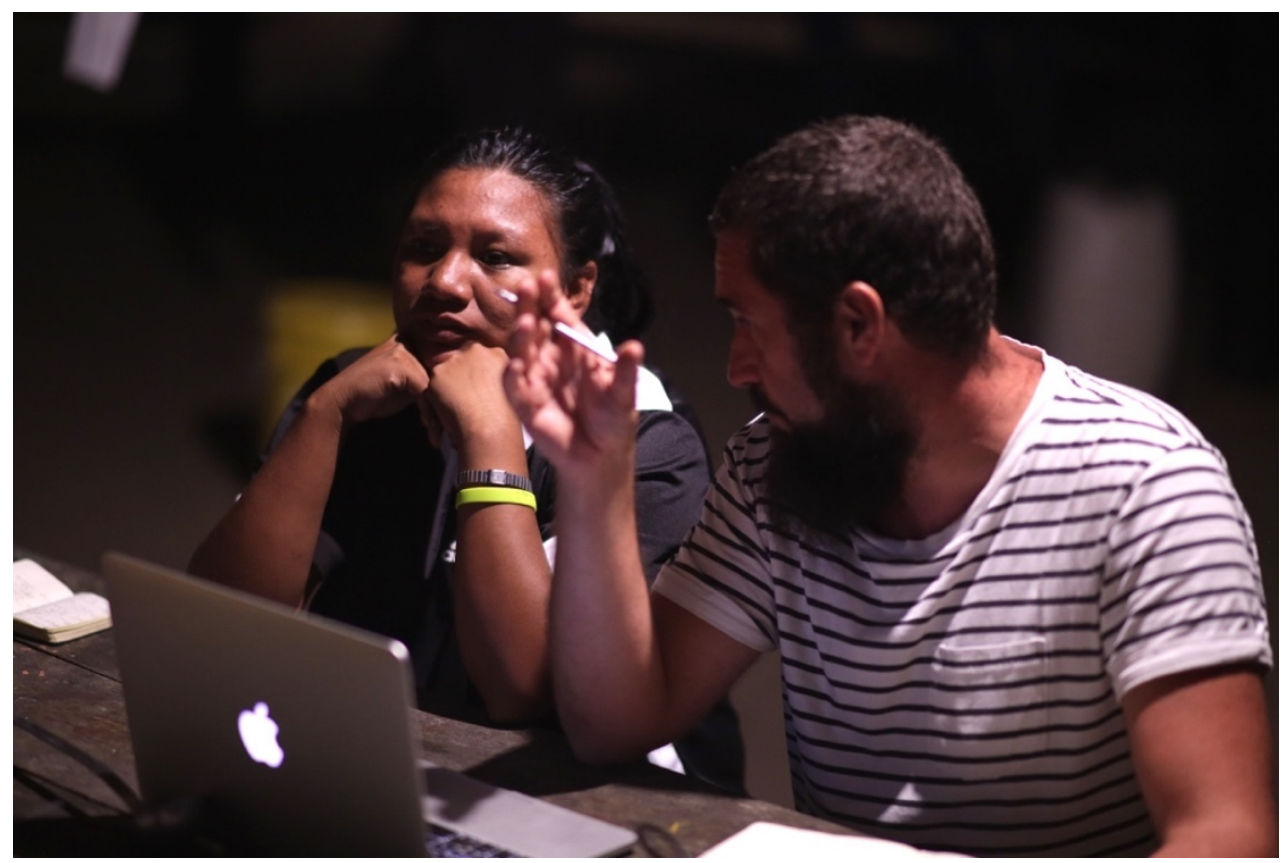

Figure 1. Travail d'écriture avec Sylvana au village. Image le GdRA (Rulhes, 2020, p.3).

Elle devait initialement y jouer ses propres rôle et texte. Suite à une promesse mal comprise et au motif de calendriers ainsi rendus incompatibles, ce souhait largement partagé par toute l'équipe de création fut finalement empêché ${ }^{3}$. Mais Selve reste une œuvre issue de Sylvana et dans laquelle elle prend la parole par son texte, ses images filmées, ses photographies et ses dessins qui font vivre la scénographie théâtrale. Sylvana y évoque sa vie et son peuple wayana, qui a connu ces cinquante dernières années une transformation fulgurante parfois brutale. La voix de Sylvana se mêle dans le texte de la pièce à celle de Selve, être chtonien féminin dont les mots sont portés par l'actrice Bénédicte Le Lamer, forêt de pensée capable de dire, quelque part entre un éco-féminisme de sorcière ${ }^{4}$ et l'anthropologie d'Eduardo Kohn. Comme si la forêt parlait en Sylvana, comme si Sylvana parlait en sa forêt, comme si Sylvana parlait en Bénédicte. Au cours de la création, à ma demande, plusieurs membres de la famille Opoya ont abordé les questions de la souveraineté villageoise et territoriale au prisme de l'orpaillage illégal et de la pollution du fleuve au mercure. Ces éléments ont intégré

\footnotetext{
${ }^{3}$ Le long récit rocambolesque de cet empêchement mériterait à lui seul un texte sur les joies, les enjeux et les affres du théâtre participatif et de témoignage. J'ai fait le choix ici de dispenser le lecteur de ce feuilleton dans lequel ont joué plusieurs personnes et institutions.

${ }^{4}$ On pense bien sûr à la figure de la sorcière et à ses multiples formes traversant la pensée éco-féministe. Pour certains passages de ce texte, j'ai proposé à Bénédicte Le Lamer de s'inspirer d'une prosodie de la magie et de la performativité, de l'incantation et de la prophétie. Elle incarne une forêt qui parle, un point de vue féminin lyrique, post-apocalyptique, survivant.
} 
la pièce de théâtre et ont influencé sa dramaturgie. L'élaboration de la pièce et l'enquête de terrain qui l'a précédée ont fonctionné comme un dispositif de recueil de parole favorisant peut-être l'expression de questions environnementales chez divers habitants de Taluhwen, prises de parole plurielle que cette contribution entend mettre en lumière. Ici, l'anthropologie a joué un rôle central, et les méthodes d'enquête par l'entretien long, biographique, le recueil d'enregistrements divers et le corpus scientifique écrit au sujet des Wayana ont joué un rôle essentiel, comme dans toutes les pièces du GdRA.

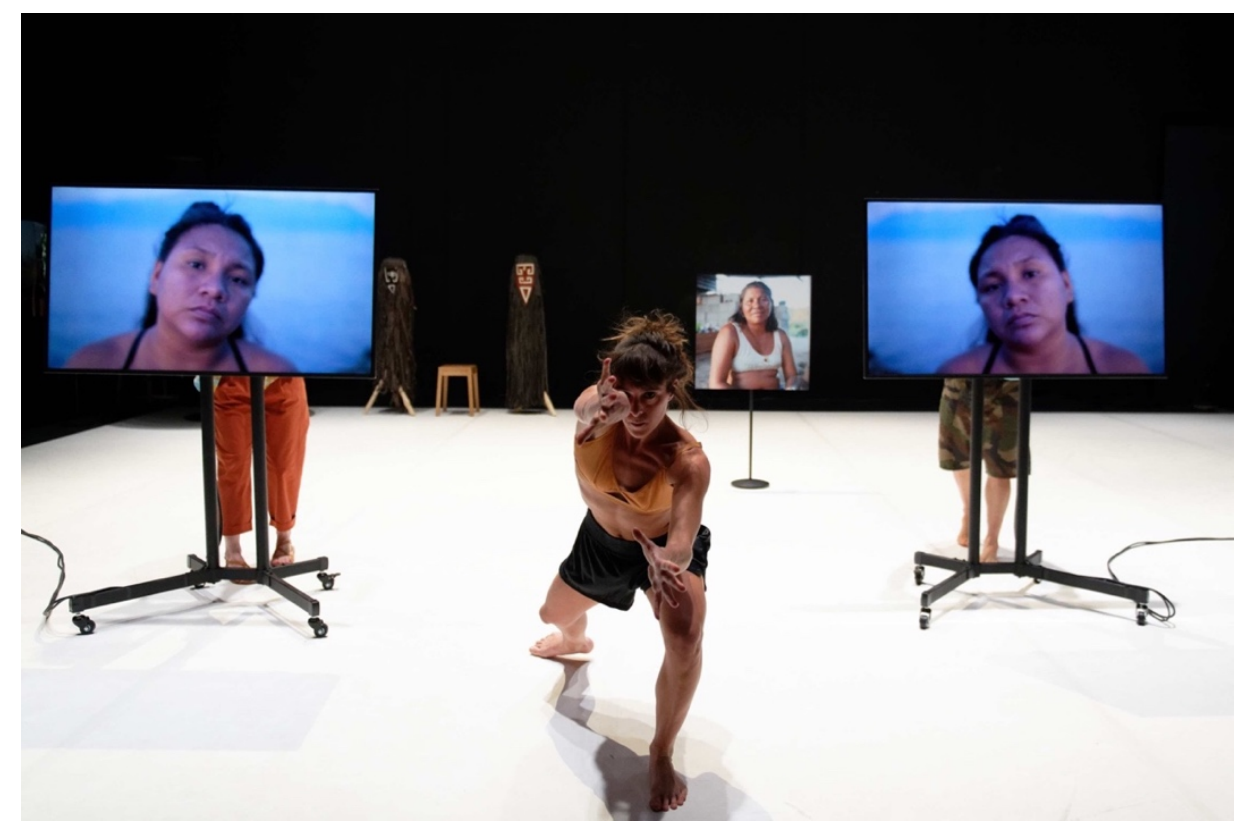

Figure 2. L'orpaillage illégal évoqué sur scène, Sylvana sur les écrans, Chloé Beillevaire dansant, Linia Opoya sur la photo centrale. Image Nathalie Sternalski le (Rulhes, 2020, p.5).

\section{Sylvana Opoya, être forêt}

Sylvana est membre de la famille du fondateur du village Taluhwen Opoya, dont on peut aussi trouver le nom écrit Taluen, Taluwen, Talhuwen, etc. Son oncle Aimawale qui participe à l'élaboration de la scénographie de Selve est le chef ou tipatakem du village depuis 2018. II est un artiste reconnu en Guyane comme en métropole pour sa production de maluwana ou "ciel de case ". II en détient le savoir-faire de son grandpère Opoya et de son grand-oncle Kuliyaman. Aima a développé sa propre technique (mais il connaît aussi la méthode ancienne du noircissement au feu et au charbon) : sur fond noir à la peinture acrylique sont remplies aux pigments naturels terreux et ferrugineux les surfaces de motifs cosmogoniques tracées au couteau, qui créent donc de légers volumes sur le bois. Pour Selve, il crée un maluwana impressionnant d'un mètre soixante-quinze de diamètre, le plus grand à sa connaissance, et il y intègre, à ma demande, un motif du danseur tamok (Duin, 2014). 
L'objet sera suspendu en cage et nez de scène, surplombant les premiers rangs des salles dans lesquelles il sera installé.

Le grand-père de Sylvana, Paranam, est le héros du livre Parana le petit indien, réalisé par la photographe Dominique Darbois et l'auteur Francis Mazière au début des années 1950. Darbois avait aussi consacré un livre photographique à l'arrière-grandpère de Sylvana, Janamale, qui avait lui aussi fondé un village sur les rives du Litani, affluent du Maroni en Guyane. En 2009, c'est cette histoire de famille que reprendra le documentariste Daniel Schweizer dans son film sur la pollution à l'orpaillage Dirty Paradise et dans lequel jouera Paranam lui-même maintenant devenu tamo, un ancien à la nombreuse descendance. Sylvana elle-même, Linia sa tante, Kindi sa sœur, Etume son oncle, Tasikale son beau-frère, ont déjà participé à l'élaboration d'œuvres photographiques, livresques, filmiques, dans lesquelles s'expriment des revendications autochtones ayant trait à la défense d'un mode de vie appartenant au paysage, à la sauvegarde d'un territoire soumis à l'extractivisme illégal et à la pollution au mercure, aux difficultés intergénérationnelles, au suicide de la jeunesse, aux influences des religions du livre et de l'évangélisme, etc (Géry, Mathieu, Gruner, 2014 ; Dewever-Plana, 2017). Enfin, Aimawale et sa mère Malilu furent parmi les informateurs privilégiés d'anthropologues, d'ethnolinguistes, de documentaristes. Aimawale a contribué à plusieurs ouvrages (Chapuis, Rivière, 2003 ; Fleury, Opoya, Aloiké, 2016). II fut l'un des inspirateurs, assistants, traducteurs, conseiller de l'œuvre magistrale de l'anthropologue Jean Chapuis consacrée aux Wayana.

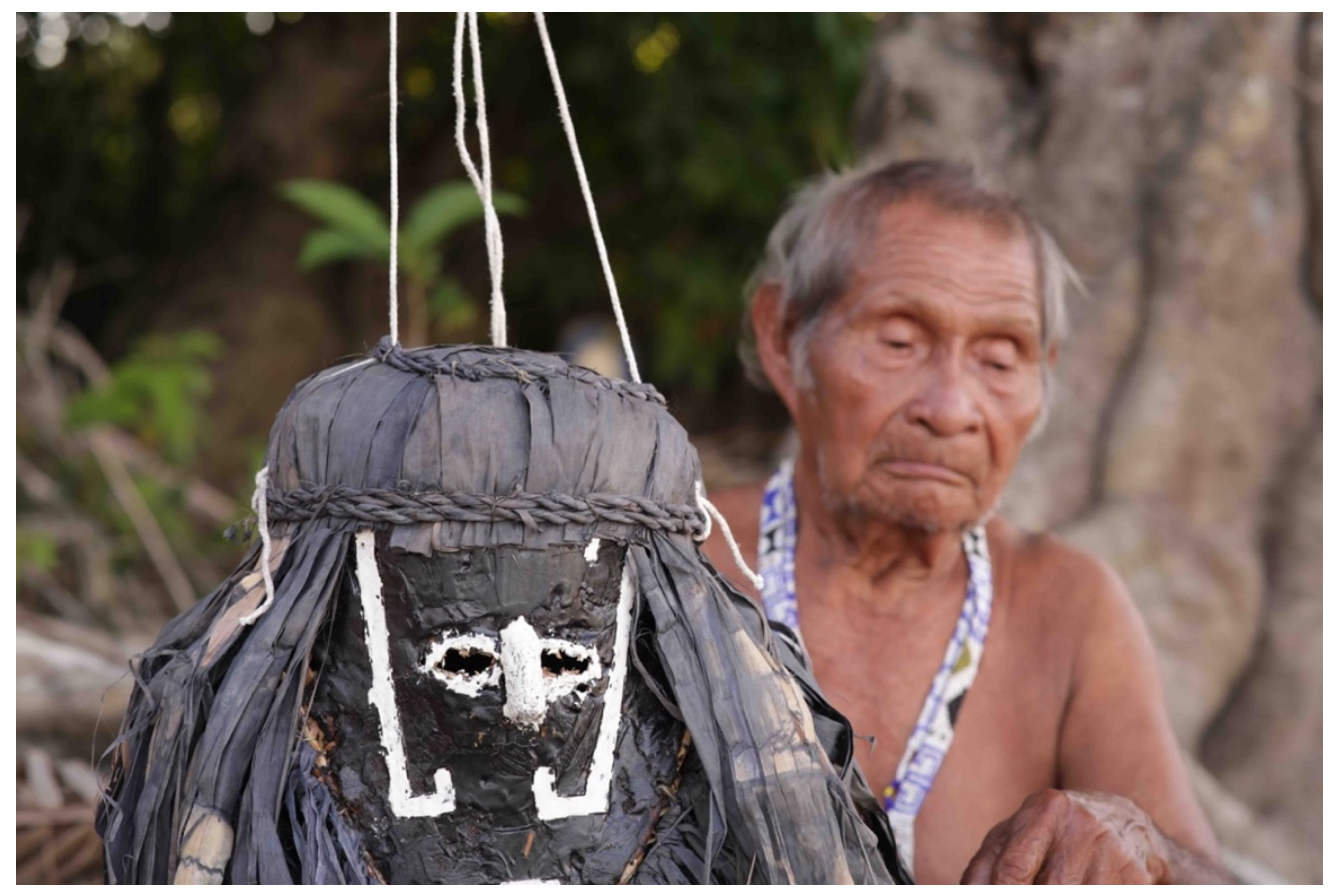

Figure 3. Tukanu, chaman, le grand-père de Sylvana. Il a notamment informé les anthropologues Jean Chapuis ou Renzo Duin. Image le GdRA Hélène Canaud (Rulhes, 2020, p.7). 
Depuis de longues années donc, de nombreux membres de la famille Opoya sont habitués à rencontrer des Palasisi, des Blancs, administrateurs, prêtres, politiciens, anthropologues, représentants d'ONG, inspecteurs du rectorat, artistes, écologues, écologistes, botanistes, missionnaires, instituteurs, etc. Ils travaillent avec eux, informent et s'informent, échangent, guident contre rétributions ou pas, sympathisent parfois, créent de profondes amitiés, participent à des projets d'envergure, collaborent à des missions anthropologiques et muséographiques, se séparent, oublient, se disputent et espèrent, attendent et provoquent, créent de profondes inimitiés. Ils sont en relation avec «le monde des Blancs », sur le mode d'une ethnicité interactionnelle productrice d'un « eux » et d'un «nous ». Ainsi même les jeunes gens comme Sylvana opposent un "nous les Wayana », " nous au village », " la Guyane de l'intérieur " à un « eux/vous les Palasisi », " ceux de Cayenne », " ceux du littoral » et, plus loin, " ceux de la Métropole ». Les plus anciens, mais cela semble récent depuis deux à trois générations, opposent très clairement « le monde moderne » à " la tradition ». Ces tensions sont présentées en continuité et sans exclusive, les personnes les moins âgées insistent sur le fait que le monde est en mouvement, et qu'aujourd'hui les Wayana sont tous allés au moins une fois sur le littoral guyanais ou à Paramaribo au Suriname pour affaire en ville. II y a encore vingt ans quelques détenteurs de savoirs oraux racontaient de mémoire d'autres mobilités et métissages lointains ou récents, entre ethnies, clans et tribus voisines, ennemies ou alliées, venues du Brésil, du Suriname ou de la Guyane française, autour des monts Tumuc Humac, entre les fleuves Paru, Jari, Litani et Maroni.

Selve est une pièce de théâtre écrite avec une jeune femme " autochtone " wayana mais dont certains membres influents de la famille maternelle se disent " métis " wayana upuliu, okomëjana et opakwana. Du côté paternel les ascendances sont apalaï, wayana, avec une présence des Aluku (groupe Noirs-Marrons ou Bushinenge descendants d'Africains ayant fui et combattu les plantations esclavagistes de la Guyane hollandaise devenue Suriname). La mémoire orale fait état à tous les stades générationnels de ces alliances commerciales et guerrières entre Boni Aluku et Wayana, et de nombreux chercheurs ont fait l'histoire de ces relations interethniques (Dupuy, 2011). Cette ascendance n'était pas connue jusque-là pour Sylvana et dans la famille Aiawali de Tukanu, et elle semble liée à un accueil après une fuite plutôt qu'à une relation matrimoniale.

Sylvana est l'une des rares femmes wayana ayant passé le baccalauréat en Guyane, peut-être la première me dit-elle, ainsi qu'au journal Médiapart en 2019, de façon plus assurée (Briswalter, 2019). Elle est plurilingue, parle le wayana, le français, plusieurs 
langues dont le sranan tongo qu'elle n'hésite pas à appeler le « taki taki » ${ }^{5}$, comprend la langue de son beau-frère kali'na, qui appartient, comme le wayana, à la grande famille linguistique karib. Sylvana a étudié à l'école de Taluhwen puis au collège et au lycée de Maripasoula. Pensionnaire, elle ne rentrait que rarement chez elle. Elle a poursuivi sa scolarité à l'Université de Kourou sur le littoral, où elle a suivi des études en lettres modernes. Ce passage à l'apprentissage sur le littoral est raconté par beaucoup de jeunes Wayana comme une étape complexe à laquelle tous ne parviennent pas à s'adapter. De fait, Sylvana n'a pas poursuivi. Elle est rentrée au village, a trouvé un poste d'intervenante en langue maternelle (ILM) à Taluhwen et se forme aujourd'hui auprès du rectorat de Guyane en souhaitant devenir maîtresse ou directrice d'école.

Sylvana est passionnée par la littérature, le théâtre, le cinéma, la diversité des langues, les séries télénovelas, le volley-ball, la musique, les images télévisuelles et la culture populaire et médiatique caribéenne. Elle s'intéresse aussi mais de plus loin aux riches pratiques artistiques wayana : conte et façons de dire, chant, musique, danse, tissage, artisanat de verroterie, vannerie, plumasserie, peinture corporelle au genipa et au roucou, sculpture, peinture, etc.

Elle fut de la dernière génération de jeunes wayana vivant en internat au home indien catholique de Maripasoula avant sa fermeture en 2012. Fondés par la préfecture et administrés par le clergé, les homes indiens de Guyane étaient des pensionnats accueillant les jeunes Amérindiens poursuivant leurs études loin de leurs villages (Armanville, 2012). Sylvana voue un profond respect pour Sœurs Maxime et Emilia, les dernières directrices du home de Maripasoula. J'ai pu longuement filmer en entretien ces deux femmes à Cayenne. Elles m'ont exposé leur point de vue quant à leur mission : protéger et accompagner les jeunes issus des communautés amérindiennes, leur apporter réconfort et enseignement et, ce faisant, insister pour qu'ils parlent le français plutôt que leur langue maternelle, inviter les garçons à se couper les cheveux, favoriser pour tous l'apprentissage du monde moderne et du travail plutôt que de "rester enfermé dans des codes traditionnels ", suggérer « l'intégration » et « l'adaptation ». Lorsque je leur parle de la richesse culturelle et ethno-scientifique des Wayana, elles font valoir une approche misérabiliste, évoquent le chômage et la pauvreté, les épidémies de suicide, l'alcoolisme et les violences conjugales des peuples forestiers de Guyane, qui justifient leur accompagnement et leur intercession bienveillante. Quand j'ai rencontré Sylvana pour la première fois, c'était dans un de ces pensionnats de mission catholique à Cayenne.

\footnotetext{
${ }^{5}$ Ensemble de langues créoles empruntant des bases lexicales à l'anglais et au portugais et parlées sur les fleuves ouest de la Guyane. La dénomination « Taki taki » est jugée péjorative et trop indifférenciée par les chercheurs, mais largement utilisée par les usagers.
} 


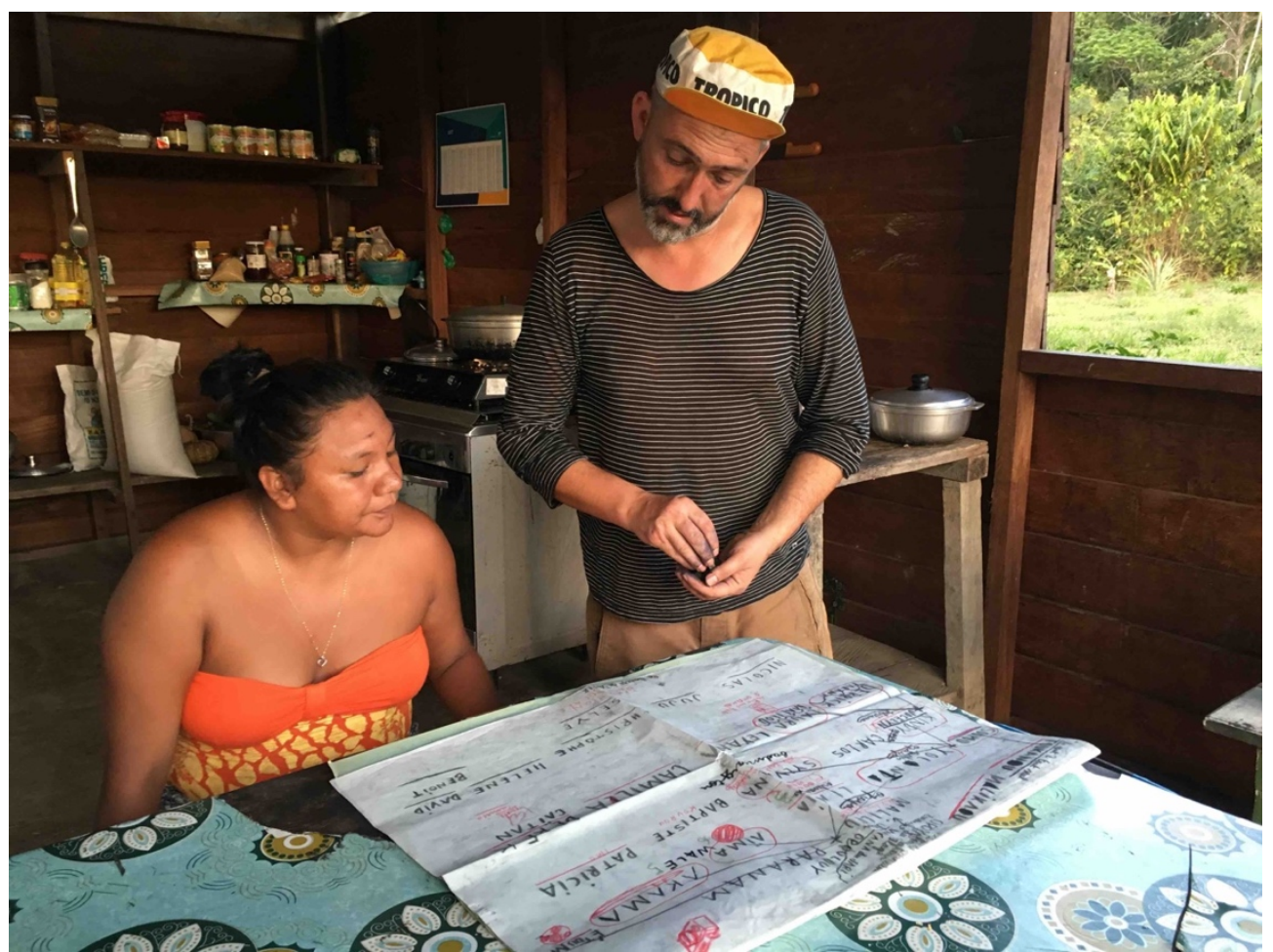

Figure 4. Sylvana indique sa généalogie au dessinateur Benoît Bonnemaison-Fitte. Image Julien Cassier le GdRA (Rulhes, 2020, p.10).

Pour autant Sylvana ne s'intéresse que très peu à la religion catholique qu'elle ne pratique pas et dont elle ne connaît pas les grands principes. Comme quasiment tous les habitants de Taluhwen, elle fut évangélisée quelques temps, convertie de façon plus ou moins consentie, mais plusieurs événements familiaux l'ont profondément attristée et mise en colère quant au prosélytisme protestant évangéliste. Elle est donc en conflit déclaré contre l'évangélisme et le protestantisme qui auraient joué, selon elle, un rôle néfaste dans plusieurs suicides au village, dont celui de son frère (Archimbaud, Chapdelaine, 2015). Ce dernier, il y a trois ans, avait fondé une église évangéliste à Taluhwen et construit en partie de ses mains l'édifice qui occupe encore le cœur d'une zone de village, à quelques pas du tukusipan, le grand carbet communautaire Wayana. Sylvana aime dire parfois comme son oncle Aima : " Je ne crois pas au dieu des Blancs, les Wayana ont leurs propres dieux. " Aima a refusé de rentrer dans les églises en Métropole lorsque je l'ai invitée en France.

Les habitants de Taluhwen racontent comment dans les familles les entrées et sorties de la religion évangéliste se font au gré de vagues successives, sont discontinues, offrent des temps de radicalisation, de syncrétisme, d'abandon. Sylvana se dit avant tout concernée par les jolok wayana, les esprits, Kuyuli le démiurge, Kailawa le héros guerrier, et par les pratiques animistes de sa communauté qu'elle cherche à comprendre. 
Du côté paternel en effet, Sylvana est la petite-fille d'un pijai fameux, un chaman craint, qui vit isolé sur les rives du Litani côté Suriname. II se dit au pays qu'il est ou fut très puissant, capable de tuer, intercesseur des âmes et des invisibles, et très compétent pour guérir et attaquer par «flèches d'esprit » ou jolok pïle. Lorsque nous filmons longuement Tukano, il raconte sa vie et sa pratique passée dont il se dit aujourd'hui certes dépositaire, mais incapable de mettre en œuvre car trop âgé. II évoque des batailles anciennes et des luttes armées à mort dont il fut un acteur important. II fabrique pour la pièce de théâtre en cours un tamok, un masque wayana sans doute lié à l'ancienne danse du pono, et que l'anthropologue Renzo Duin fait remonter aux premiers contacts entre Wayana et conquistadors de l'expédition d'Orellana (Duin, 2014). Sylvana est fière de sa filiation chamanique et de son ascendance de chefs, fière de la présence sur scène d'objets wayana aussi forts que les tamok et le maluwana, mais elle n'hésite pas non plus à moquer ma passion pour les « histoires et les choses anciennes » et me fait découvrir Ximena Duque ou Karina Kapoor, des héroïnes de séries colombienne et bollywoodienne. Le quotidien de Sylvana est pluriel, il ne se résume pas à une alternative à deux termes entre espoir et nostalgie, entre deux rives et deux mondes. II s'agit plutôt d'un plurivers personnel profus et continu aux arborescences, opportunités et brisures nombreuses. Aujourd'hui Sylvana se forme pour devenir institutrice, était toute proche de jouer en tant qu'actrice d'une pièce de théâtre figurant des éléments de sa vie en Métropole, publie avec moi le texte de cette forme théâtrale chez un éditeur reconnu en la matière, a déjà joué avec notre ami commun Nicolas Pradal dans Anuktatop, un documentaire fiction largement montré ${ }^{6}$. Elle souhaite toujours apprendre l'espagnol et aimerait favoriser la transmission de la culture wayana et des arts à Taluhwen, et dit ne pas renoncer à pouvoir jouer encore du théâtre ou du cinéma.

\section{«Quand ils nettoient leur or, ils ne font pas attention à nous ! "}

À Cherbourg dans les salles de répétitions de la Brèche, lors d'une discussion avec le public suite à un travail d'étape, dans le cadre d'une rencontre éditoriale organisée par la revue $Z$ au sujet des "trésors et conquêtes " en Guyane ( $Z 12$ la Revue $Z$, 2018), aux questions de la journaliste, Sylvana prend fermement la parole contre l'orpaillage illégal et la montagne d'or, pour la préservation du mode de vie forestier et fluvial des Wayana, en carbets ouverts, sans électricité et surtout sans voiture, avec la chasse, l'abattis et la pêche. Elle parle de la pollution et des « marchandises des Blancs ».

\footnotetext{
${ }^{6}$ Après huit ans de voyages en pays wayana, considéré comme membre du village de Taluhwen, le réalisateur Nicolas Pradal fut le guide précieux et diplomate du GdRA dans ses voyages en Guyane. Nicolas (Pradal, Selvini, 2016).
} 
Le lendemain, elle est très heureuse que nous mangions ensemble un kebab au fastfood et souligne avec une pointe d'ironie sa performance de la veille. Elle me dit aimer en villes métropolitaines ou au village la nourriture industrielle surgelée, qu'elle trouve parfois meilleure et certainement plus pratique à consommer que le produit de la chasse ou de la pêche. Nous rions. Je lui rappelle que l'un de ses noms wayana est Alimina l'anguille, qui se faufile entre les rochers du fleuve sans perdre son chemin. Kawui son compagnon qui voyage parfois avec nous en métropole pour les répétitions de création, ne supporte pas quant à lui la nourriture trop transformée et revendique pleinement, toujours très humblement et sans effusion, ses talents de pêcheur et de chasseur, sa faculté à vivre en forêt. Les Wayana sont différents les uns des autres. Il est bon de le rappeler. Ils offrent à l'autochtonie autant de prismes qu'il y a de personnes, et se fédèrent différemment des Kali'na, vivant sur le littoral de la Guyane, qui ont créé des organisations politiques autochtones dès les années 1980, mais dont la langue se fragilise plus grandement que celle des Wayana.

Au sujet de l'orpaillage illégal et comme pour l'évangélisme, la situation est complexe, avec de jeunes Amérindiens qui peuvent participer un temps à cette activité, puis la dénoncent fermement, avant d'y plonger à nouveau pour intégrer ensuite un mouvement politique anti-orpaillage.

Dans ce maelström croisé, comment vivent à Taluhwen trois cent habitants, en ce lieu villageois sédentarisé il y a quarante ans et scolarisé il y en a trente, où des pirogues monoxyles, des carbets aux toits en feuille, des vanneries d'agriculture, de chasse, de pêche et de cuisine issues de plusieurs décennies voire des siècles de transmissions et d'innovation sur ce territoire amazonien, côtoient des bidons de gasoil en plastique, des touques de navigation en polyéthylène et des pirogues en aluminium, des groupes électrogènes, des paraboles et des écrans plasma ? Où l'eau courante tout au long de la journée et le réseau électrique pour tous ne sont toujours pas intégralement installés ou arrivent à peine en 2019, malgré les demandes incessantes de certains habitants depuis plus de vingt ans ? Quel rôle y joue la pollution au mercure, au plomb et à la boue qui « salit » le fleuve à vue d'œil, handicape des nouveaux nés et des enfants, pose la question de l'usage quotidien de l'eau'? « L'eau est sale » disent les habitants.

À Taluhwen le fleuve est un lieu de repos, de jeu, de rencontre et de discussion. II est avec la forêt une matrice importante de la vie villageoise. Le fleuve permet de se laver, de se rafraîchir, de nettoyer les vêtements et la vaisselle. « Le fleuve c'est la salle de

\footnotetext{
${ }^{7}$ À ce sujet on peut visionner le documentaire édifiant de Daniel Schweizer, Dirty Paradise, 2009. On peut y voir filmés Paranam lui-même et Etume le plus jeune oncle de Sylvana ; Aimawale et Tasikale y prennent aussi la parole. Pour l'évocation d'études récentes sur la pollution au mercure et au plomb accompagnées de chiffres suivre Laramée de Tannenberg, 2018. Pour une étude datant de 2007 lire « Le mercure en Guyane. Risques sanitaires et enjeux de santé publique ", Numéro thématique du BASAG, Institut de Veille Sanitaire, CIRE Antilles Guyane. Pour une étude alarmiste de 1998 voir Grasmick, 1998.
} 
bain, la baignoire, la buanderie, le lave-vaisselle, les W.C., la piscine, le gardemanger... » nous dit en riant Kindi qui connaît bien les modes de vie forestier et citadin. Il est des courants du fleuve qui servent de toilettes et on s'y éloigne de la berge pour soulager le corps. Le fleuve est pourvoyeur de nourriture et de bienfaits, il est un lieu d'affirmation, un refuge à la fois public et domestique, on y démontre des amitiés, des appartenances et des affinités familiales, on y voit et on y est vu, y vivre à proximité est une marque de qualité ${ }^{8}$. Le fleuve est le lieu de la relation avec les autres communautés, du commerce et de l'histoire récente des Wayana.

Quelle est au fleuve la frontière entre le propre et l'impropre, le propre et le public, le propre et le fluide ? Entre la pollution et la propreté ? Le mercure des garimperos s'y mélange avec les liquides vaisselle et les lessives des Wayana, les excréments se mixent avec l'eau, la terre, la boue, le limon, les poissons et les crustacés. Que peut en écrire Sylvana dans son texte ou en dire Aimawale dans les entretiens filmés que nous réalisons ensemble afin de préparer sa participation à la scénographie de Selve ? Des préoccupations « environnementales » ou "écologistes » teintent-elles les contributions d'Aimawale et de Sylvana à la pièce Selve ? Si oui, d'où viennentelles, de nous, d'eux ? Il est très difficile de savoir ici qui parle exactement d'écologie, tant le monde vécu semble un entrelacs d'influences diverses où les personnes se baignent malgré tout, respirent quoiqu'il en soit, continuent de manger le poisson que les médecins et les scientifiques de passage leur conseillent de ne plus consommer. Qui parle, des autochtones ou des écologistes Blancs?

Le grand nombre de personnes qui prennent la parole au nom du fleuve, de la terre et de la forêt et contre la pollution moderne des blancs en Guyane, parfois largement assumée par certains Amérindiens eux-mêmes, est toutefois significatif en pays wayana et dans toutes les communautés amérindiennes. II y a ici une forte congruence entre savoir vivre forestier et engagements écologistes, et l'ancien maire de Camopi Réne Monerville, ou Jean-Paul Fereira, maire de Awala-Yalimapo, respectivement Amérindiens Teko et Kali'na, eurent ou ont encore des responsabilités en construisant politiquement des listes électorales écologistes. On pense aussi aux engagements de Brigitte Wyngaarde, Ti'iwan Couchili, Cécile Couyouri, cheffes coutumières ou actives politiquement sur des listes écologistes. À Taluhwen, Aimawale dit pour la pièce de théâtre, que les Amérindiens n'ont rien à apprendre des philosophes Blancs de l'écologie, et que "le système nature » n'existe pas en pays Wayana (Glowczewski, 2015). Un texte très proche de celui qui est donné ci-dessous fut enregistré en vidéo est utilisé dans Selve. II est visible en suivant ce lien : https://vimeo.com/295874601.

\footnotetext{
${ }^{8}$ Voir à ce sujet les travaux nombreux de Jean Chapuis et sa très fine description de l'histoire du rapport au fleuve chez les Wayana, avec leur sortie progressive de la forêt profonde, pour pacifier les relations, installer des comptoirs commerciaux et entretenir des échanges politiques.
} 
II n'y a pas de pensée amérindienne ${ }^{9}$. C'est une histoire de blancs. II n'y a pas de pensée amérindienne de la nature, c'est faux, on ne peut pas inventer ça. Ce que nous disons, et ce que disait mon grand-père, c'est qu'il n'y a pas d'environnement autour de nous. L'environnement, la nature, tout ça, et bien nous sommes dedans, comme des fleurs, comme un arbre, comme un oiseau, je suis un arbre. Je pense que notre maison est là, dans ces êtres. Tout individu qui se trouve ailleurs dans un milieu, crée son mode de vie bien entendu, mais à travers tous les êtres auxquels il appartient... il n'y a pas de système nature et tout ça, puisque tout se transforme entre tous les êtres. Je pense que le mode de vie Wayana est né là, dans le milieu. Nous avons un grand respect pour nous, donc pour tous les êtres de l'environnement des Blancs... et nous avons notre propre mythe qui parle de ce que vous appelez l'environnement, où nous sommes dedans, transformés en lui et avec lui. Je respecte bien les écologistes, mais ils sont un peu en retard je pense ! Ils ont déjà fait ce qu'il ne fallait pas faire, donc maintenant ils essaient de réagir ! Nous c'est l'inverse, nous on respecte, on est déjà Ecologie ! Quand tu ne sais plus chasser, quand tu ne sais plus pêcher, ne parle pas d'écologie aux Amérindiens : tu es déjà dépassé, tu es déjà à l'écart. Je peux parler parce que je vis dans ce milieu, je pêche, je chasse, j'ai des enfants, je ne veux pas qu'ils se marient avec des Français même si j'aime beaucoup certains d'entre eux, je préfère rester chez moi. C'est pareil avec les Marrons, je les aime aussi, mais si vraiment on essaie de défendre l'identité wayana, et tous ces aspects écologiques, il faut se garder soi-même, se garder nous-même. Et je le dis à tous les leaders, amérindiens ou Blancs : "Est-ce que vous avez encore un abattis ? Non ? Pourquoi tu parles d'écologie aux Amérindiens alors ? Si tu ne sais pas planter, tu ne peux pas t'exprimer sur l'abattis, tu ne connais pas ! " Je vis dans la réalité. Sans l'environnement je ne peux pas vivre. Ce n'est pas autour, c'est dans moi, je suis dedans. L'environnement il peut vivre sans moi. L'environnement ne m'appartient pas. C'est moi qui suis son objet, son affaire. Alors quand on dit " la pollution " aujourd'hui, moi je dis attends : la colonisation n'est pas finie. Toutes les pollutions qu'on voit là, les poubelles, les déchets, nous nous battons pour la cause du fleuve, pour qu'il n'y ait plus de poubelles... le fleuve était clair et les Boni et les Wayana vivaient en paix, maintenant c'est sale et ils se disputent pour savoir qui va nettoyer. Même si je ne crois pas en l'écologie, enfin au système nature, je le vois à ma façon. Les milieux aquatiques sont déjà créés, la forêt est déjà créée, donc on ne touche pas, on ne pollue pas, c'est concret. Ce n'est pas parce qu'on est moderne, qu'on a des toilettes, une douche, qu'on dort sur un lit - les Wayana vont vers ça - qu'il faut oublier la terre. Tu travailles, tu as un diplôme, c'est bien. Mais la vraie richesse, celle de quatre-vingt pour

\footnotetext{
${ }^{9}$ Dans la pièce de théâtre Selve, par choix artistique, politique, anthropologique, toutes les prises de paroles de Sylvana ou d'Aimawale se font en wayana sur-titré ; dans le livre La Guerre des Natures, selon les mêmes critères de choix, une bonne part des textes de Sylvana apparaissent en wayana écrit, accompagné du CD de traduction. Ici, pour des raisons de place je ne fais figurer que des traductions françaises de ces extraits d'entretien.
} 
cent des Wayana, c'est celle du jardin, de l'abattis, du fleuve, de la terre. Vingt pour cent sont pauvres, ils ne savent plus faire l'abattis, pêcher et chasser, et c'est eux qui te parlent de pollution ! La vraie richesse c'est l'abattis, ça c'est un salaire, une liberté. Tu plantes, tu manges. Si tu ne plantes pas, tu peux aller chez les copains une fois, deux fois, mais au bout d'un moment... Beaucoup disent : " je préfère acheter de la marchandise ". Même moi j'achète d'ailleurs. Mais si le commerce s'en va, s'il ferme, comment tu fais, comment tu achètes ? Voilà comment tout se perd, tresser l'arouman, modeler la terre, planter. Et après on vient nous parler de pollution.

Lorsque je me retrouve en situation d'entretiens avec Sylvana pour préparer son texte dans la pièce, j'opte pour une méthode biographique dans laquelle nous essayons de créer une linéarité narrative. Sylvana y est à l'aise dans l'art de l'autoportrait, mais elle raconte aussi au fil d'analogies thématiques et emprunte aux façons de dire traditionnelles. Le fleuve revient toujours au centre. Lorsque nous abordons « le mode de vie wayana », elle parle de " la pollution moderne » qui concerne essentiellement l'eau et les rives du fleuve : rejets de déchets et d'emballages, de fluides, de gasoil, de mercure, de plomb, notamment autour des chantiers d'orpaillage et des supermarchés " chinois " qui animent la vie commerciale des rives côté Suriname. Elle développe un passage sur l'extractivisme aurifère illégal dans lequel elle insiste sur les combats menés par les Wayana et sa tante Linia pour lutter contre les chercheurs d'or clandestins. Elle souligne aussi son rejet des voitures et son attachement à la pirogue. Elle ne veut pas de piste ou de route jusqu'à Taluhwen, sous prétexte que cette construction abîmerait la forêt et que « les Wayana en voiture ne seraient plus des Wayana ». Pourtant, elle espère passer un jour son permis de conduire à Cayenne. Lorsque nous choisissons ensemble les passages d'enregistrement qu'elle souhaite voir contribuer à son texte définitif, elle propose que figure ce paragraphe relatif à la fièvre de l'or, " parce que c'est un problème important au village ». Sur ce lien vidéo on peut voir Sylvana lire son texte en Wayana au cours d'une répétition https://vimeo.com/295150240 :

Cela fait longtemps que l'orpaillage illégal existe chez nous, c'est le problème principal du fleuve. C'est depuis Pitimaina que les orpailleurs nous polluent. L'eau est terriblement sale, je ne le supporte pas, à tel point que je ne m'y lave pas toujours ! Pourquoi ? Mes yeux brûlent, mes cheveux s'abîment, l'eau est trop sale ! Quand ils nettoient leur or ils ne font pas attention à nous ! Ils laissent le mercure qui s'écoule au fleuve, puis les poissons le mangent, nous mangeons le poisson et nous tombons malades. Nous avons tout un tas de maladies à cause du mercure. Pourquoi les orpailleurs nous détruisent ? Quand la situation s'est empirée, les Wayana ont mis des barrages à Cayode. ${ }^{10}$ II y avait beaucoup de barrages, il fallait $\mathrm{y}$

\footnotetext{
${ }^{10}$ Cayode est un village wayana situé sur les rives du Tampok, sous-affluent du fleuve Maroni, à quelques heures de pirogue de Taluhwen. Les évaluations de la pollution mercurielle ont montré que ce village était le plus
} 
donner sa carte d'identité... Tu devais prouver ton identité à l'aller et au retour de Cayode. Aujourd'hui l'eau de Cayode est tellement sale ! C'est vraiment triste... II y a des malades à cause de la pollution ! II y a des malformations à cause du mercure, des enfants qui ne peuvent pas parler, des personnes qui ont des handicaps, c'est terrible, c'est ainsi qu'arrivent les nouveaux nés ! Je me souviens de cette histoire, les militaires n'étaient pas encore là, les Wayana ont décidé d'agir, le grand-chef des Wayana a dit aux orpailleurs " arrêtez ! ". Si on attrapait les orpailleurs, ils se faisaient tabasser. Les Wayana fouettaient les orpailleurs avec des branches. Ma tante Linia se battait. Elle se bat encore contre l'orpaillage et pour défendre notre terre.

Ici il est bien clair que c'est Sylvana qui parle. Mais aurait-elle spontanément donné ce texte lors de nos très longs entretiens, si je n'avais pas évoqué le témoignage que sa sœur Kindi avait livré à des journalistes sur ces questions-là ? Qui, des autochtones ou des écologistes Blancs, a lancé l'alerte en premier ? Vraiment, est-ce si important que cela de le savoir ? Ici en Amazonie, la parole autochtone est depuis trop longtemps subordonnée, mal entendue, et peut sans doute éclairer les préoccupations des écologistes et des philosophes de l'anthropocène. Cinq cent ans d'apocalypse, cela forme l'écologie des pratiques, le sens de la survie, le sens de la vie, de l'adaptation et de l'hybridation. Les Wayana n'ont pas attendu les représentants d'ONG pour lutter contre les garimperos.

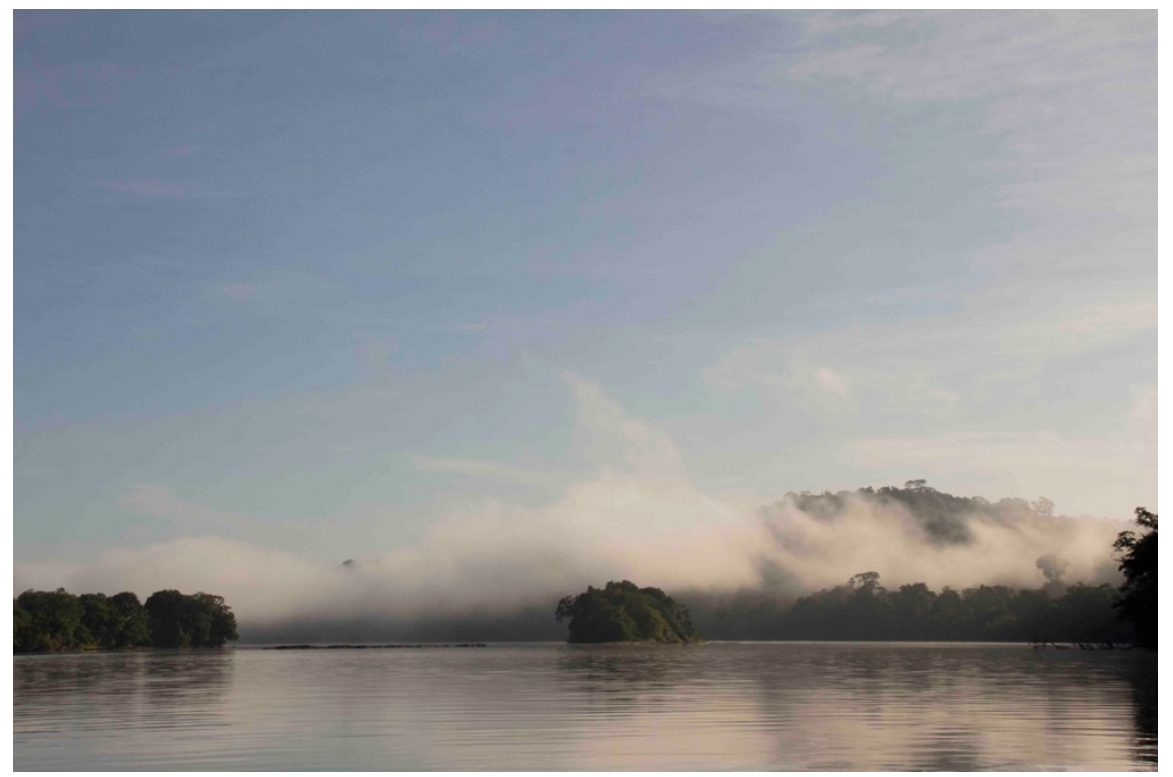

Figure 5. Chez Sylvana, le fleuve au matin, où l'on se lave avant la journée (Rulhes, 2020, p.18).

contaminé du pays Wayana. Mélanie Aliman'Hé, cheffe coutumière de Cayode, a souvent témoigné dans les médias guyanais contre l'orpaillage. Elle prend aussi largement la parole dans le film Dirty Paradise. Sylvana parle des barrages érigés régulièrement depuis 2006, parfois par les gendarmes, souvent par les locaux, pour empêcher le trafic fluvial des orpailleurs. Plusieurs coups de feu furent échangés, et de nombreux récits d'accrochage ou d'arrestations violentes entre orpailleurs et Wayana circulent à Taluhwen, Cayode, Elahé. Le soir à la veillée, la parole se déliant autour d'une bière ou d'un cachiri, la boisson locale de manioc, des récits de violences explicites ou de meurtres peuvent être entendus ou sous-entendus. 


\section{Plastique et ferraille dans les champs du Quercy}

Je ne sais plus qui m'a enseigné le fait de parler à une sauterelle ou à un gouffre. Chez nous en Aveyron sur les causses du Quercy, au cœur de terres paysannes aux traditions de petites exploitations semi-autarciques, les buttes et les combes ont des noms en occitan qui évoque des histoires anciennes et des récits récents. Le paysage parle et mes parents et grands-parents s'adressaient à lui, lo defòra, le dehors, à travers ses nombreux existants, plantes, pierres, animaux, arbres. En la terra nostra, dans notre terre, les personnes vivent en continuité avec la guêpe, dont il faut voir et aimer le mouvement pour danser avec elle et ne pas se faire piquer. Partanhem a la terra me disait souvent mon grand-père : « nous appartenons à la terre ». Enfant j'ai pu enregistrer : La terrà es totjorn la mai forta. Nautres, sem a sa rason. Es pas vertadierament la terrà nostra. Pertanhem a la terrà que rebiscola totjorn. Sem defòra, "La terre est toujours la plus forte. Nous autres, sommes à sa merci. Ce n'est pas vraiment notre terre. Nous appartenons à la terre qui revit toujours. Nous sommes dehors ". De là vient le théâtre que j'essaie de fabriquer aujourd'hui en équipe. Lorsque j'ai pu lire un jour Philippe Descola et les anthropologues ou philosophes du drame écologique, je n'ai pu m'empêcher de sentir en ces lignes le souffle de l'esprit de ma famille paysanne du dehors. Et lorsque j'enregistre Aimawale en Guyane, je retrouve cette volonté d'appartenance à la terre, cette façon de dire du dehors, dans le dehors, au sein même d'un écoumène limité et fragile.

Une langue parlée en un lieu, sur une terre native, qui dit le paysage, et qui se laisse dire par lui. Sans doute mes ascendants étaient-ils des " autochtones », de ceux qui dépendent de l'histoire d'un territoire et de ses lieux-dits, non écrits, légendés à l'oral plutôt que sur une signalétique. De ceux qui arrivent en un lieu et qui veulent le dire. Aujourd'hui les militants occitanistes parlent une langue métisse, entre le français et la lyrique des troubadours, entre la langue sauvage et vernaculaire des paysages et la langue juridique et bureaucratique du français, entre les mouvements de la littérature moderne et contemporaine et " le patois » du villageois qui ne s'écrit pas. Ils ont mangé la langue de l'ennemi et l'utilisent à tout va. Ils jouent d'antagonismes et de contradictions.

Dans les champs et les rivières de la paysannerie, le plastique, les pesticides et la ferraille ont ponctué les paysages. Les natifs eux-mêmes ont parfois participé à ces mutations, acceptant plus ou moins les influences et les pressions extérieures, administratives et bancaires, étiques et esthétiques. Certains ont livré bataille et continuent de défier la grande transformation imposée depuis les années cinquante. Aujourd'hui avec l'agroécologie et la permaculture, de jeunes agriculteurs venus d'ailleurs ou d'ici reprennent les techniques paysannes "à l'ancienne », s'intéressent aux pratiques "des vieux », cherchent les variétés et les cépages qui peuplaient les 
champs avant l'apparition de la monoculture. Une nouvelle génération de militants occitans cherche un peuple perdu, développe une ethnogenèse originale et s'approprie le terme d'autochtonie, avec ses territoires, ses fractures, ses endémies, ses migrations et ses inventions, quitte à méconnaitre ou à détourner l'histoire politique et internationale du mot $^{11}$, forgée au loin entre les revendications des peuples et terres en danger et les bureaux de l'ONU.

En pays wayana l'autochtonie s'anime comme un être échevelé. On s'invente chaman pour plaire au Blanc et à l'anthropologue, on crée de nouveaux motifs artistiques pour satisfaire une curiosité et une demande grandissante venue de "l'extérieur », on établit des rapports avec un ordinateur, on fait de la politique à Maripasoula, on travaille au Rectorat, on cache des informations jugées trop importantes, certains objets rares ou phrases puissantes ne sont dévoilés qu'après une longue relation de confiance, les noms des derniers " véritables chamans " ne sont pas dits, on manifeste une pluralité d'attachements, on valorise un savoir-faire céramiste ancien, on participe aux Journées Autochtones jugées par certains trop folkloriques ou dictées par la culture de masse télévisuelle, on organise des jeux inter-villages comme dans le Rouergue ou le Quercy, on amplifie la musique de tambour ou de flûtes et partout se fait entendre un rap en langue amérindienne teinté de musique afro-caribéenne.

S'il arrive donc souvent qu'une Wayana vous parle avec les mots d'une évangéliste prosélyte de la modernité et de la bienséance consumériste, il se peut aussi qu'elle s'adresse à son interlocuteur avec les mots d'une responsable d'ONG environnementale ou d'une garde responsable du Parc Amazonien de Guyane. D'ailleurs, les Wayana commencent à briguer des postes dans les institutions françaises. Comme les Occitanes du Rouergue, Sylvana se laisse traverser par un flux pluriel d'influences, qu'elle anime et oriente en retour, en fonction de ses choix de vie personnels et singuliers. Elle cherche son devenir autochtone, sa participation active au village, affine en son territoire ses choix de vie et de subsistance, et ne cesse de déplorer "l'eau sale " du fleuve qui lui brûle les yeux. Un midi au restaurant japonais à Toulouse, elle me dit émue que les Wayana vont disparaître. La langue restera peut-être avec un certain esprit, mais le carbet, la pirogue, la pêche et la chasse disparaîtront. Et même la langue s'affaiblira. Je lui demande ce qu'elle en pense au fond. Elle me répond en riant : " Mais des personnes seront vivantes, des descendants de Wayana habiteront à Paris, Miami, Cayenne... ils seront vivants ! ».

\footnotetext{
11 « Je suis même pas né là, mais je suis autochtone. J'ai réappris la langue du pays, je cultive à l'ancienne, j'ai rencontré tous les vieux pour qu'ils transmettent. Certains se moquent. Mais moi je veux vivre ici, avec la logique alternative, et personne ne doit me dire qui je suis et où j'habite, je suis autodéterminé autochtone, je suis de cette combe et j'y accueille qui veut venir. C'est ça mon autochtonie, une paysannerie anarchiste qui veut s'implanter dans la terre, vivre dans la terre. "Camille, paysan à côté de Saint-Clair de Margues dans l'Aveyron, locuteur occitan au lexique plus large que le mien, pourtant né ici de cette terre et de cette langue.
} 
Dans Selve, Sylvana comme Aimawale ont influencé par leurs mots et leurs gestes artistiques le récit qui s'est constitué avec eux. Ils participent directement à une œuvre théâtrale, dans laquelle, au cœur de la personne de Sylvana qui se déploie dans ses associations plurielles, ils manifestent une volonté souverainiste d'auto-détermination personnelle avant tout. Parfois, cette dernière aborde très clairement et fermement l'ambition de maintenir une souveraineté sur le territoire wayana, en réaction à la marchandise et à la pollution des Blancs, avec l'administration française, tantôt en alliance, tantôt en défiance avec la République et la Métropole. Cependant, pour Sylvana comme pour Aimawale, les propos sont nuancés et contrastés, comme les choix de vie sont en partie différents et singuliers. Sylvana assume avec joie ce qu'elle refuse de voir comme des contradictions, et développe une vitalité toute forestière qui doit s'accommoder de la mort, quelque part entre putréfaction et germination, entre disparition et vie, dans la transformation. Aimawale tient un propos de chef de village soucieux de préserver avant tout un mode de vie auquel il est profondément attaché, et dans lequel là aussi la transformation animiste et la continuité entre les existants joue un rôle central.

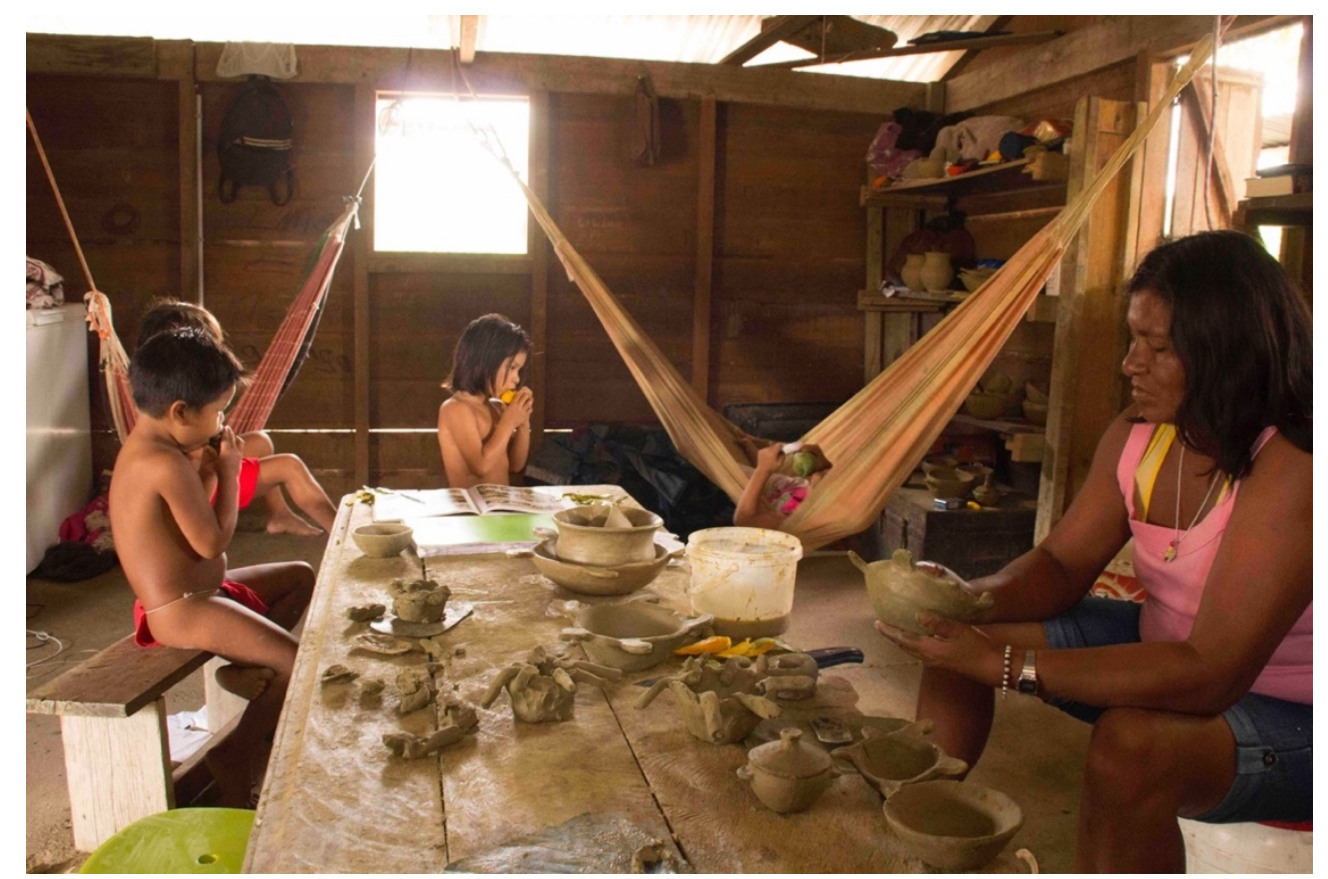

Figure 6. Poterie wayana au village avec Linia Opoya et ses jeunes apprentis. Sylvana est très attentive à la transmission vers les enfants. Image Hélène Canaud le GdRA (Rulhes, 2020, p.22). 


\section{References}

Armanville, F. (2012). Les homes indiens en Guyane française : pensionnats catholiques pour enfants amérindiens. 1942/2012. Université d'Aix-Marseille.

Archimbaud, A., Chapdelaine, M-A. (2015). Suicides des jeunes Amérindiens en Guyane française : 37 propositions pour enrayer ces drames et créer les conditions d'un mieuxêtre. Rapport établi par les Parlementaires en mission auprès de Madame la ministre des Outre-mer.

Briswalter, M. (2019). Le défi de l'éducation nationale dans le Haut-Maroni. Médiapart. https://www.mediapart.fr/journal/france/100819/le-defi-de-leducation-nationale-dans-lehaut-maroni?onglet=full

Chapuis, J., \& Rivière, H. (2003) Wayana eitoponpë. (Une) Histoire (orale) des Indiens Wayana. Cayenne : Ibis Rouge Édtitions.

Dewever-Plana, M. (2017). D'une rive à l'autre. Paris, Blume.

Duin, R. (2014). Historical complexity of myth: in search of the genesis of the whip-dance whereby Wayana dance in imitation of Tamok (Eastern Guiana Highlands). In Boletim do Museu Paraense Emílio Goeldi. Ciências Humanas, 9(3), set.-dez, 741-772. http://dx.doi.org/10.1590/1981-81222014000300013

Dupuy, F. (2008). Wayana et Aluku : les jeux de l'altérité dans le haut Maroni. In Collomb, Gérard et Jolivet, Marie-José (éds), Histoires, identités et logiques ethniques. Amérindiens, Créoles et Noirs Marrons en Guyane (pp. 165-201). Paris : CTHS.

Fleury, M., Alupki, T., Opoya, A., Aloïké, W. (2016). Les Wayana de Guyane française sur les traces de leur histoire. Revue d'ethnoécologie, 9. https://doi.org/10.4000/ethnoecologie.2711

Géry, Y., Mathieu, A., Gruner, C. (2014). Les abandonnés de la République. Paris: Albin Michel.

Glowczewski, B. (2015). Au cœur du soleil ardent : la catastrophe selon les Aborigènes. Communications, 1(96), 53-65.

Grasmick, C. (1998). La pollution mercurielle liée à l'orpaillage en Guyane : contamination des systèmes aquatiques et impact sanitaire chez les Amérindiens du Haut-Maroni. Journal d'agriculture traditionnelle et de botanique appliquée, 40(1-2), 167-179.

Laramée de Tannenberg, V., (2018). Toujours trop de plomb et de mercure chez les Guyanais. Journal de l'environnement, 5 février.

http://www.journaldelenvironnement.net/article/toujours-trop-de-plomb-et-de-mercurechez-les-guyanais,90337

Pradal, N., \& Selvini, P. (2016). Anuktatop, la métamorphose. France:Chercheurs d'autres.

Rulhes, C., le GdRA. (2019). La Guerre des Natures, Lenga, Selve. Besançon: Les Solitaires Intempestifs.

Schweizer, D. (2009). Dirty Paradise. France, Switzerland : Maha Production, Horizon Films. Z12, la Revue Z. (2018). Guyane, Trésors et conquêtes. Montreuil. 
Christophe Rulhes was born into a peasant and Occitan family. A graduate in anthropology from the Ecole des Hautes Etudes en Sciences Sociales, Christophe practices music and singing and writes and directs plays based on investigation and testimony - where words are heard from different perspectives, bodies, and languages - for the GdRA, a multidisciplinary artist collective. He constantly seeks to dialogue with people, while understanding that all life choices are singular and there is a multitude of paths people can take. His research and practice asks the questions: What do the arts and the humanities, the ordinary and theater have in common? What narratives are they creating and for what audiences are they produced? 